\title{
Silencing of Jagged1 inhibits cell growth and invasion in colorectal cancer
}

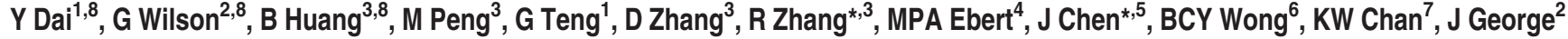 \\ and L Qiao*,2
}

Dysregulated Notch signaling has a critical role in the tumorigenesis. Jagged1, a Notch ligand, is overexpressed in various human cancers. Recent studies revealed the involvement of Jagged1 in colorectal cancer (CRC) development. These basic studies provide a promising potential for inhibition of the Notch pathway for the treatment of CRC. Herein, we aimed to investigate the consequences of targeting Jagged1 using shRNA on CRC both in vitro and in vivo to test their potential to inhibit this key element for CRC treatment. We found that downregulation of Jagged1 with lentiviral Jagged1-shRNA resulted in decreased colon cancer cell viability in vitro, most likely mediated through reduced cell proliferation. Importantly, Jagged1 knockdown induced $G_{0} / G_{1}$ phase cell cycle arrest, with reduced Cyclin D1, Cyclin $E$ and c-Myc expression. Silencing of Jagged1 reduced the migration and invasive capacity of the colon cancer cells in vitro. Furthermore, colon cancer cells with knockdown of Jagged1 had much slower growth rate than control cells in a xenograft mouse model in vivo, with a marked downregulation of cell proliferation markers (PCNA, Ki-67, and c-Myc) and metastasis markers (MMP-2 and MMP-9). These findings rationalize a mechanistic approach to CRC treatment based on Jagged1-targeted therapeutic development.

Cell Death and Disease (2014) 5, e1170; doi:10.1038/cddis.2014.137; published online 10 April 2014

Subject Category: Cancer

Colorectal cancer (CRC) is the third most common malignancy in males and second most common in females worldwide. ${ }^{1}$ The highest incidence of $\mathrm{CRC}$ is found in Australia, New Zealand and North America, and the morbidity is rapidly increasing in Eastern Asia and Eastern Europe. ${ }^{2,3}$ About $40-50 \%$ of all patients with CRC will present with metastasis either at the time of diagnosis or develop distant relapses after therapy, and the median overall survival of metastatic CRC is less than 2 years. ${ }^{4}$ Current therapeutic options for advanced CRC, such as chemotherapy and radiotherapy, only have limited efficacy and can barely improve patient survival. A better understanding of the molecular mechanisms involved in CRC development and progression is imperative for the improvement of therapeutic approaches that can benefit patients with CRC.

The Notch signaling has an important role in cell growth and differentiation, which affects the development and function of many organs. ${ }^{5,6}$ Notch receptors (Notch1-4 in humans) are a group of transmembrane proteins in many cell types. Binding to their ligands (Jagged1, 2 and delta-like ligand (DLL) 1, 3, and 4 ) on the surface of neighboring cells leads to the cleavage of Notch receptor by $\gamma$-secretase and subsequent release of the Notch intracellular domain (NICD). As the constitutively active domain of the Notch receptor, NICD can translocate to the nucleus, where it binds to and forms a complex with the transcriptional regulator termed CBF-1-Su $(\mathrm{H})$, and LAG-1 (CSL) or RBP-Jê, leading to the displacement of co-repressors previously bound to CSL and recruitment of co-activators. The co-activators then induce expression of the target genes, such as the hairy and enhancer of split (Hes) and Hes-related repressor protein (Hey) families. Accumulating evidences indicate that dysregulated Notch pathway has a critical role in the progression of several malignancies such as prostate cancer, breast cancer, glioma and head and neck cancers. Furthermore, high expression levels of several key members of the Notch cascade such as Notch1 and Jagged1

\footnotetext{
${ }^{1}$ Department of Gastroenterology, Peking University First Hospital, Beijing, China; ${ }^{2}$ Storr Liver Unit, Westmead Millennium Institute, Department of Medicine and Western Clinical School, The University of Sydney, Westmead, NSW, Australia; ${ }^{3}$ Research Center of Basic Medical Sciences and Department of Immunology, Key Laboratory of Immune Microenvironment and Diseases of Educational Ministry of China, Tianjin Medical University, Tianjin, China; ${ }^{4}$ Department of Medicine II, Klinikum Rechts der Isar, Technical University, Munich, Germany; ${ }^{5}$ School of Biomedical Sciences, The University of Queensland, Brisbane, QLD, Australia; ${ }^{6}$ Departments of Medicine, The University of Hong Kong, Pokfulam, Hong Kong and ${ }^{7}$ Departments of Pathology, The University of Hong Kong, Pokfulam, Hong Kong

${ }^{*}$ Corresponding author: R Zhang, Research Center of Basic Medical Sciences and Department of Immunology, Key Laboratory of Immune Microenvironment and Diseases of Educational Ministry of China, Tianjin Medical University, Tianjin 300070, China. Tel/Fax: + 8622 83336563; E-mail: rongxinz@yahoo.com

or J Chen, School of Biomedical Sciences, The University of Queensland, Brisbane, QLD 4072, Australia. Tel: +61 733651409 ; Fax: +61 733651766 ; E-mail: j.chen4@uq.edu.au

or L Qiao, Storr Liver Unit, Westmead Millennium Institute, Department of Medicine and Western Clinical School, The University of Sydney, Westmead, NSW 2145, Australia. Tel: +61 29845 9132; Fax: + 6129845 9103; E-mail: liang.qiao@ sydney.edu.au

${ }^{8}$ These authors contributed equally to this paper.

Keywords: colorectal cancer; Notch signaling; Jagged1; targeting; xenograft tumors

Abbreviations: Apex, purinic-apyrimidinic endonuclease-1; CDK, cyclin-dependent kinase; CRC, colorectal cancer; DLL, delta-like ligand; Hes, hairy and enhancer of split; Hey, Hes-related repressor protein; L-Jagged1-shRNA, lentiviral Jagged1-shRNA; MMP, matrix metalloproteinase; NICD, notch intracellular domain; PCNA, proliferating cell nuclear antigen

Received 05.1.14; revised 18.2.14; accepted 26.2.14; Edited by G Raschellá
} 
are associated with increased progression and metastatic potential, recurrence and a poorer overall survival. ${ }^{7-11}$ Recently, Lu et al. ${ }^{12}$ showed endothelial cells could secrete Jagged1 and mediate the activation of Notch signaling in CRC, leading to increased expression of Hes1. Kim et al. ${ }^{13}$ demonstrated that Jagged1 was a mediator of a purinic-apyrimidinic endonuclease-1 (apex)-induced CRC progression.

The role of Notch signaling in the development of CRC has attracted wide attention over the past few years. ${ }^{14}$ Animal studies have shown that Notch is active in intestinal crypts and adenomas in $\mathrm{APC}^{\mathrm{Min} /+}$ mice $^{15}$ Moreover, deletion of one Jagged1 allele is sufficient to significantly reduce the size of tumors in the APC mutant background concomitant with a reduction in the amount of active Notch $1 .{ }^{16}$ It was shown that Notch signaling is strongly activated in primary human CRC and has an important role in the initiation and progression of $\mathrm{CRC}$ through the regulation of the main cellular functions associated with tumorigenesis, such as apoptosis, proliferation, angiogenesis, and cell migration. ${ }^{17-19}$ Thus, the Notch pathway has been regarded as an attractive target for CRC therapy. ${ }^{20,21}$ One approach currently being explored in clinical trials is blocking the cleaving process of Notch receptors with $\gamma$-secretase inhibitors. These agents have shown the therapeutic benefit for CRC. ${ }^{22}$ Although $\gamma$-secretase inhibitors are valuable tools for delineating the function of Notch cascade, they were found to be not specific for Notch signaling and were associated with various adverse effects such as gastrointestinal toxicity and liver injury. ${ }^{23}$ Notch downregulation is fundamental in driving secretory cell differentiation in the normal intestinal mucosa ${ }^{15,24,25}$ thus, inactivation of the Notch signaling causes secretory cell metaplasia, and results in inflammatory response. ${ }^{25}$ The efficacy of this class of compounds needs exploring, but the relative lack of target specificity suggests that new and more specific strategies targeting this pathway should be pursued. A recent study has demonstrated that inactivation of DLL1 and DLL4-mediated Notch signaling results in loss of intestinal proliferating progenitors due to conversion into postmitotic goblet cells, but inducible deletion of Jagged1 has no overt phenotype. ${ }^{26}$ Moreover, expression of Jagged1 was restricted to enteroendocrine cells or undetectable in the mucosa of the human small and large intestine, respectively. In contrast, increased expression was found in human colon tumors. ${ }^{20}$ These data suggest that Jagged1 may be a more specific target molecule for developing new therapy against CRC.

As most studies have focused on the effects of inhibiting the Notch receptor and its downstream signaling, specific inhibition of Jagged1 in CRC has not been fully explored. In this study, we aimed to determine the efficacy of specific targeting of Jagged1 as a therapeutic approach for CRC. In vitro and in vivo models were utilized to investigate the effects of Jagged1 knockdown on cell viability and invasion in CRC.

\section{Results}

High expression of Jagged1 in human CRC tissues was associated with activation of the Notch pathway. We first examined the expression of Jagged1 in 24 pairs of human colon cancer tissues and matched non-cancerous colonic mucosa by qPCR. As shown in Figure 1a, the majority
(20/24, or $83 \%$ ) of cancer tissues (tumor) exhibited higher expression level of Jagged1 relative to their corresponding non-cancerous controls (normal). Figure $1 \mathrm{~b}$ shows that the average expression of Jagged 1 mRNA was $\sim 6$-fold higher in tumor tissues than in normal tissues $(P<0.01)$. Higher expression levels $(>2.5$-fold) of Jagged1 protein in colon cancer tissues were also confirmed by immunohistochemistry (Figures 1c and d). The increased Jagged1 expression in tumor tissues was associated with activation of Notch signaling, with an increased expression of Notch target gene Hes1 (Figure 1e). These data confirm that Jagged 1 is frequently overexpressed in CRC and likely responsible for the constitutive activation of Notch signaling, implying that selectively, targeting this protein may present a novel therapeutic strategy in CRC. Therefore, we designed shRNA and packaged it into a lentiviral vector to generate lentiviral Jagged1-shRNA (L-Jagged1-shRNA), and the efficacy of this vector-mediated Jagged1 knockdown against CRC was tested in a series of in vitro and in vivo assays.

Knockdown of Jagged1 led to reduced Notch signaling activity in CRC cells. Protein expression of Jagged1, as measured by western blot, was prominent in human colon cancer cell lines HCT15, HT29, DLD1, HCT116 and SW480 (Figure 2a). When these colon cancer cells were infected with L-Jagged1-shRNA for $72 \mathrm{~h}$, there was a marked reduction in the expression of endogenous Jagged1 (Figure 2b), confirming a successful gene knockdown. Importantly, silencing Jagged1 was sufficient to significantly decrease the expression of Hes1 at both mRNA and protein levels, as exemplified in HT29 cells (Figures $2 \mathrm{c}$ and d, respectively), suggesting that the expression of Jagged1 is a general characteristic of colon cancer cell lines, and that Jagged1 knockdown causes Notch signaling activity inhibition.

Downregulation of Jagged1 induced cell growth inhibition and $G_{0} / G_{1}$ cell cycle arrest in colon cancer cells. Given the increased expression of Jagged 1 in the CRC tissues and colon cancer cell lines, we determined whether Jagged1 downregulation affected colon cancer cell proliferation in vitro. HCT15, HT29, DLD1 and HCT116 cells were transiently transfected with L-Jagged1-shRNA or scrambled-shRNA for $24 \mathrm{~h}$, and cell viability was assessed by $\left[{ }^{3} \mathrm{H}\right]$-thymidine incorporation assay $72 \mathrm{~h}$ later. As shown in Figure 3a, Jagged1 downregulation decreased cell viability by $39 \%, 58 \%, 47 \%$, and $41 \%$ in HCT $15, \mathrm{HT} 29$, DLD1, and HCT116 cells, respectively. In addition, Jagged1 knockdown cells exhibited decreased anchorage-dependent growth property. The clonogenicity was reduced by $76 \%, 62 \%$, and $85 \%$ in HCT15, HCT116, and SW480 cells, respectively (Figure 3b), indicating that downregulation of Jagged1 suppressed colon cancer cell proliferation.

To determine the mechanism by which Jagged1 downregulation may affect cell growth, cells were infected with L-Jagged1-shRNA or scrambled-shRNA, and cell cycle analysis was carried out. As shown in Figure 4b, knockdown of Jagged1 led to decrease in the percentage of SW480 cells in $S$ phase by nearly $10 \%$ and increased the $G_{0} / G_{1}$ fraction 
a

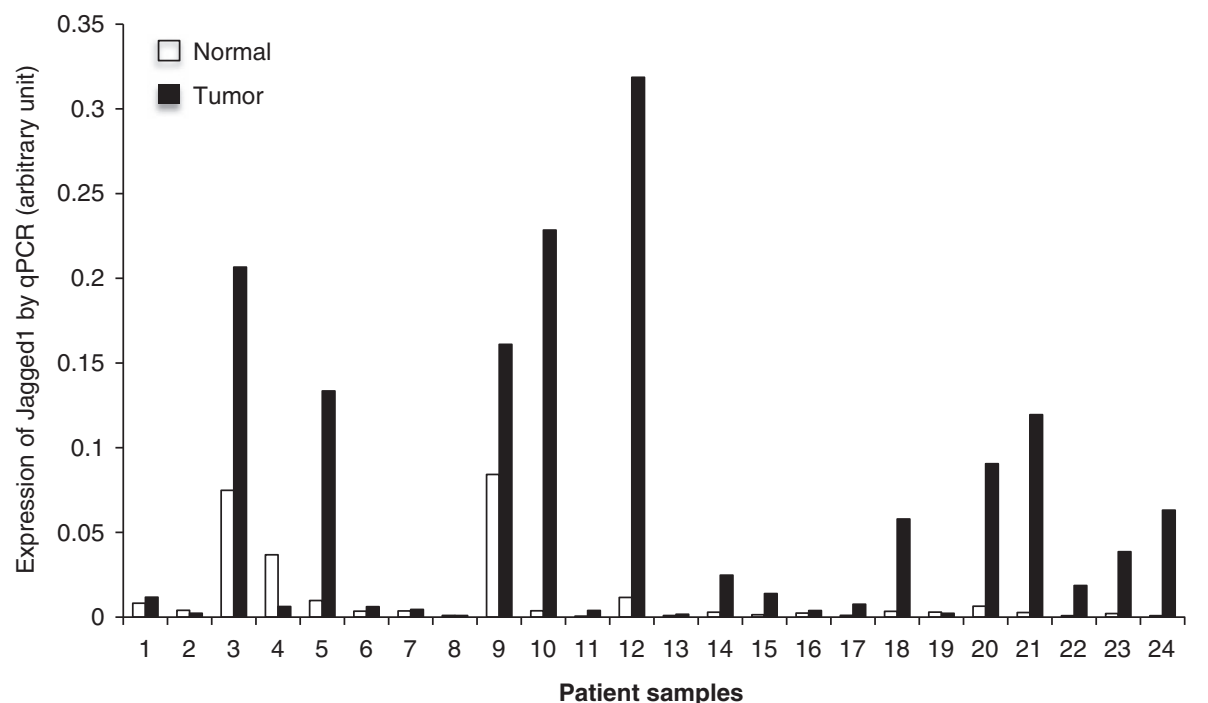

C
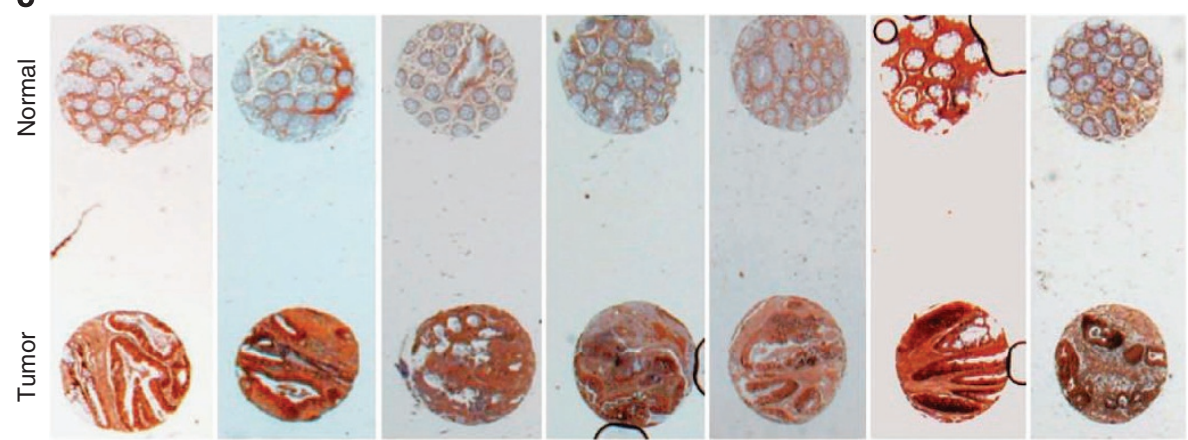
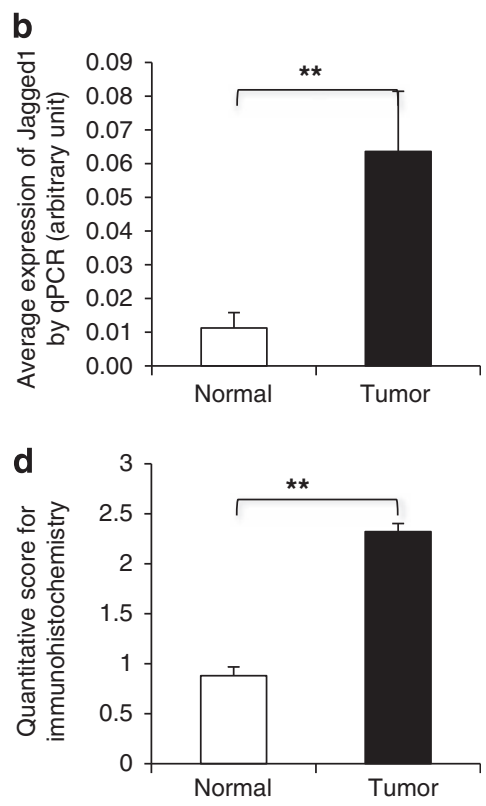

Figure 1 Expression of Jagged1 by qPCR in 24 pairs of colon cancer (tumor) and matched non-cancerous colonic tissues (normal) (a). On average, higher expression level of Jagged1 was found in tumor than in normal tissues $(n=24, \mathbf{b})$. In seven selected cases, higher expression of Jagged1 in tumor tissues was confirmed by immunohistochemistry $(n=7, \mathbf{c}, \mathbf{d})$. Expression of Notch downstream target gene Hes1was examined by qPCR in cancerous (tumor) and matched non-cancerous (normal) colon tissues (e). ${ }^{* *} P<0.01$

a

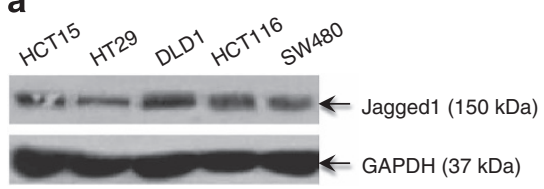

C

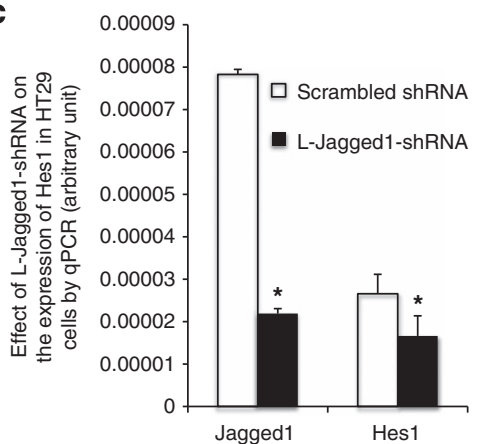

b

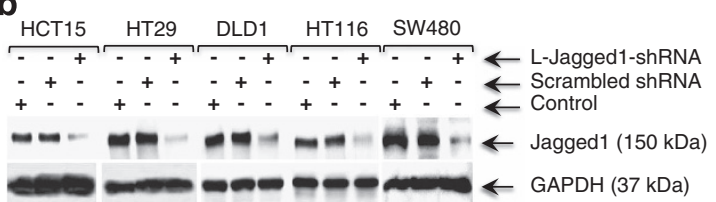

d

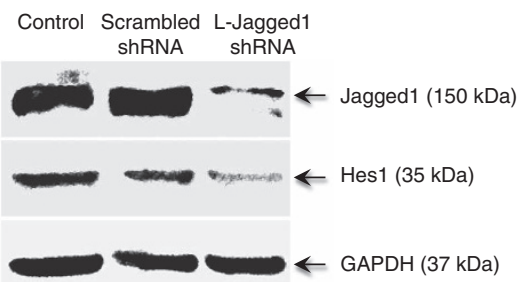

Figure 2 Expression of Jagged1 was determined by western blot in five colon cancer cell lines (a). L-Jagged1-shRNA successfully knocked down Jagged1 in these cells (b). Downregulation of Jagged1 was associated with a reduced expression of Notch downstream target Hes1 in HT29 cells at the mRNA (c) and protein levels (d). ${ }^{*} P<0.05$. The experiments were repeated at least three times 

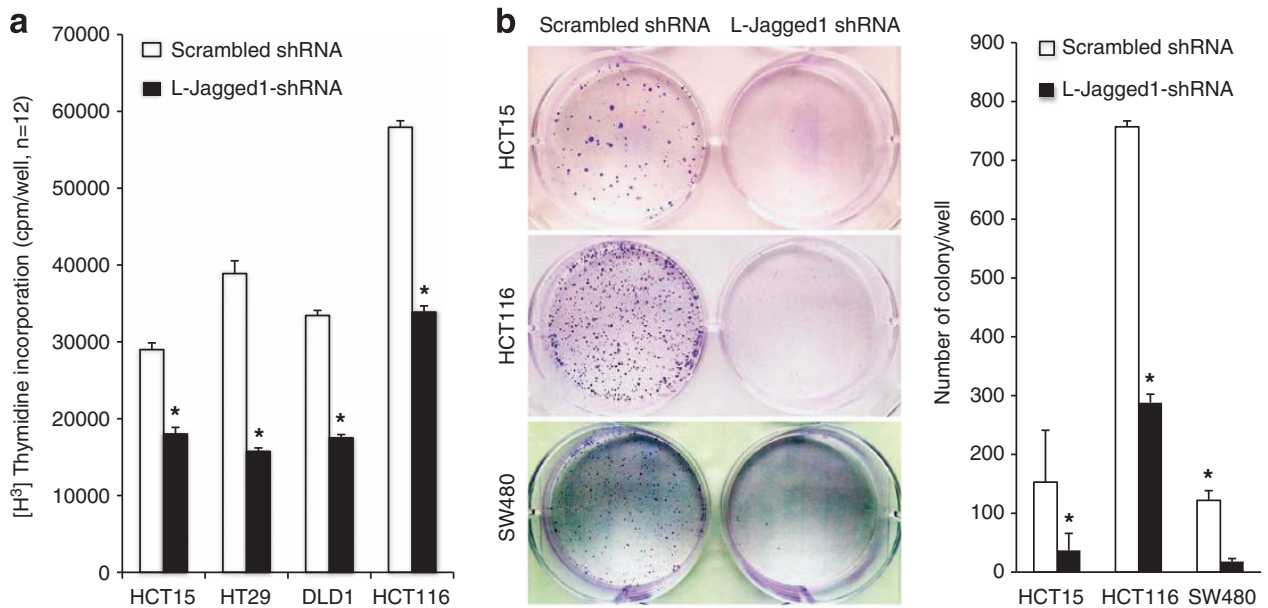

Figure 3 Knockdown of Jagged1 led to a significant inhibition of proliferation of colon cancer cells HCT15, HT29, DLD1, and HCT116 (a). Marked reduction of colony formation was observed in HCT15, HCT116, and SW480 cells transfected with L-Jagged1-shRNA (b). ${ }^{*} P<0.05$. The experiments were repeated at least three times
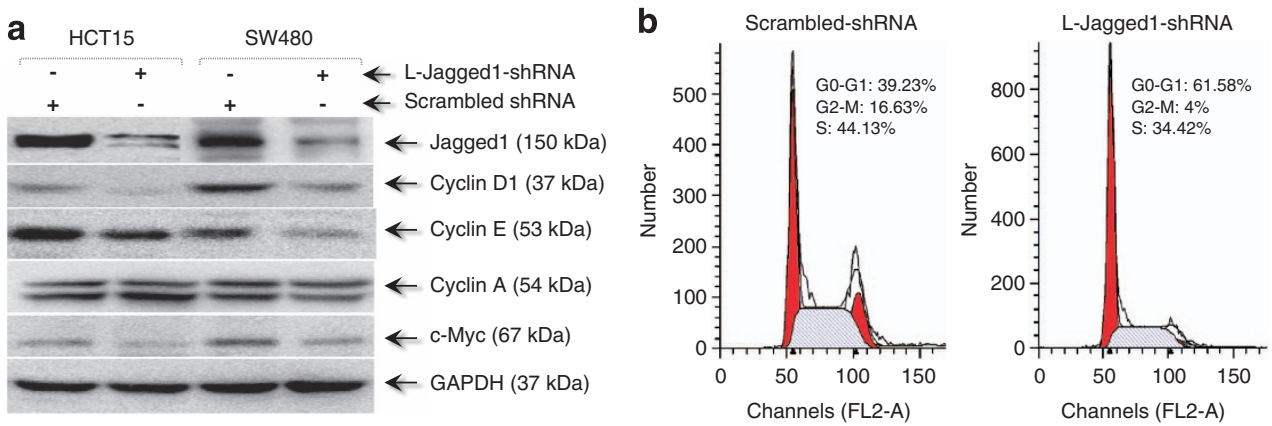

Figure 4 Expression of cell cycle-related proteins Cyclin D1, Cyclin E, Cyclin A, and c-Myc in human colon cancer cells HCT15 and SW480 after treatment with L-Jagged1-shRNA (a). Cell cycle analysis was examined by FACScan using SW480 cells as an example (b). The experiments were repeated at least three times

from $39.23 \%$ to $61.58 \%$, indicating a $G_{0} / G_{1}$ cell cycle arrest. A similar result was found in HCT15 cells (data not shown). Furthermore, cell cycle-related protein expression was assessed by western blot. Consistent with the accumulation of cells in $\mathrm{G}_{0} / \mathrm{G}_{1}$ phase, Cycline D1, Cyclin E, and c-Myc significantly decreased in L-Jagged1-shRNA-treated HCT15 and SW480 cells, whereas Cyclin A remained unchanged, compared with scrambled-shRNA-treated cells (Figure 4a). In addition, there was no significant increase in apoptosis upon Jagged1 knockdown in HCT15, HCT116, and SW480 cells as detected by AnnexinV/PI staining (data not shown). Together, these results indicated that Jagged1 silencing in colon cancer cells was associated with a block in cell cycle progression and not increased apoptosis.

Downregulation of Jagged1 decreased the migration and invasion capacity of colon cancer cells. Elevated cell migration and invasion are associated with increase in the metastatic potential of cancer cells. This may be independent of cell proliferation rates. Therefore, we studied the effect of Jagged1 downregulation on the migration and invasion of colon cancer cells. Cell migration was determined by woundhealing assay. As shown in Figure 5a, Jagged1 downregulation significantly suppressed the migration of HCT116 cells. The migration index of Jagged1 knockdown cells was decreased by $49 \%, 47 \%$, and $36 \%$ at 24,48 , and $72 \mathrm{~h}$, respectively (Figure $5 \mathrm{~b}$ ). To examine the cell invasion activity in vitro, we used transwell inserts coated with matrigel matrix. After Jagged1 knockdown, the invasiveness of HCT116 cells was decreased by $43 \%$ as compared with the control cells (Figures $5 c$ and $d$ ).

Silencing of Jagged1 suppressed the growth of xenograft tumors in nude mice. To explore the effects of Jagged1 knockdown in vivo, xenograft tumors were generated by injecting HCT15 cells stably infected with either control vector (scrambled-shRNA) or L-Jagged1-shRNA. The cells were injected s.c. into the flanks of nude mice. As shown in Figure 6a, tumors derived from HCT15 cells previously infected by scrambled-shRNA grew fast, particularly 13 days after injection. Compared with the control group, tumors derived from L-Jagged1-shRNA-infected cells grew much slower throughout the experiment, suggesting that knockdown of Jagged1 dramatically impaired the tumorigenic growth of HCT15 cells. At harvest, the tumors from L-Jagged1-shRNA-infected cells weighed much lighter than those from control cells (>8-fold) (Figure 6b). The average volume of tumors derived from Jagged1 knockdown cells showed $\sim 10$-fold reduction compared with control (Figures $6 c$ and $d)$. 
a
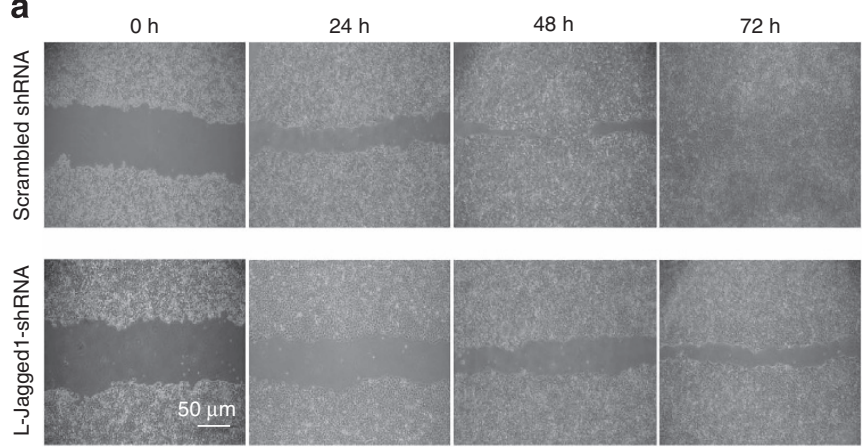

C

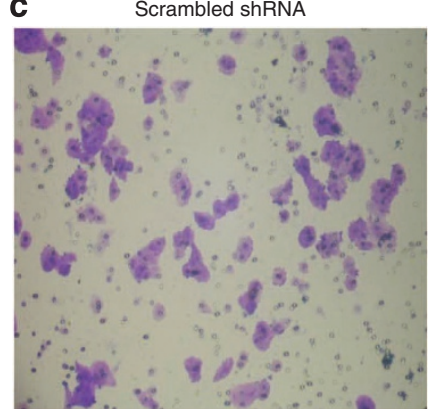

L-Jagged1-shRNA

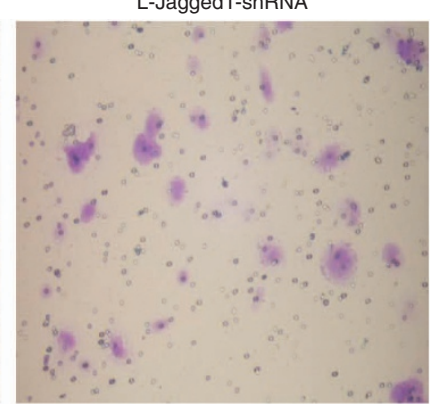

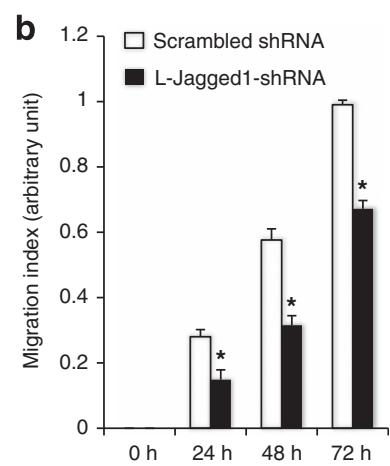

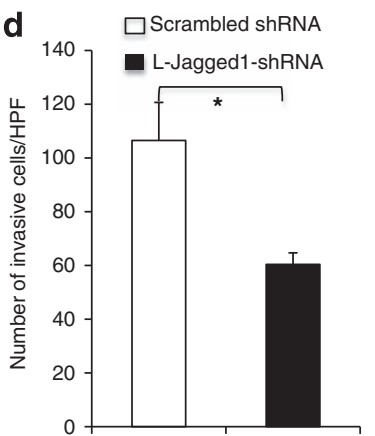

Figure 5 Knockdown of Jagged1 led to a significant inhibition of the migratory ability of HCT116 cells (a). Knockdown of Jagged1 led to a significantly slower migration at 24,48 , and $72 \mathrm{~h}$ after cells were infected with L-Jagged1-shRNA (b). ${ }^{*} P<0.05$. Similarly, inhibition of Jagged1 led to a reduced invasive ability of HCT116 cells (c, d). ${ }^{*} P<0.05$. The experiments were repeated at least three times

To further verify the antitumor effects of Jagged1 silencing, we next examined the expression of cell proliferation markers (Ki-67, PCNA, c-Myc), metastasis markers (MMP-9, MMP-2) and angiogenesis marker CD31 at the mRNA and protein levels in xenograft tumors. As shown in Figure 7a, there was a significant reduction in the mRNA expression of Ki-67, PCNA, c-Myc, MMP-9, and MMP-2 in tumor tissues. The mRNA level of CD31 in tumors was not altered upon Jagged1 knockdown. Immunohistochemical staining confirmed these findings, as exemplified by the reduced $\mathrm{Ki}-67$ and MMP-9 expression in the xenograft tumors derived from HCT15 cells infected by L-Jagged1-shRNA (Figure 7b).

\section{Discussion}

In the current study, we found that Jagged1, one of the most important ligands for the Notch signaling, is overexpressed in $\sim 80 \%$ of human CRC tissues, and is associated with a marked elevated expression of Notch target gene Hes1. Targeting Jagged1 induces growth inhibition in colon cancer cells both in vitro and in vivo, at least in part by causing $\mathrm{G}_{0} / \mathrm{G}_{1}$ phase cell cycle arrest. In addition, downregulation of Jagged1 reduces the migration and invasion of colon cancer cells, most likely involving decreased MMPs.

Notch signaling is critical for determination of cell fate within a wide variety of tissues by regulation of growth, differentiation, and apoptosis. Abnormal Notch signaling is associated with tumorigenesis ${ }^{7-11}$ and cell invasion. ${ }^{11,27-29}$ Previous studies have shown aberrant expression of the Notch pathway in CRC. ${ }^{16,20,30}$ In particular, Jagged1 was found to be overexpressed in $\sim 50 \%$ of human colon tumors, while it was undetectable in the normal colonic mucosa. ${ }^{20}$ Moreover, the levels of Jagged1 expression were correlated with tumor differentiation parameters and stages of CRC. ${ }^{30,31}$ It was found that high expression of Jagged1 is associated with poor clinical outcome in some malignancies such as breast cancer and prostate cancer, whereas another study demonstrated high Jagged1 levels were correlated with better prognosis of CRC. ${ }^{31}$ The association and prognostic value of Jagged1 in CRC remains to be investigated further. In this study, we confirmed that $>80 \%$ of CRC tissues expressed higher level of Jagged1 compared with normal colonic tissues, and that this was associated with a marked elevated expression of the Notch target gene Hes1. These data suggested that Jagged1 is likely a major ligand responsible for Notch signaling activation in $\mathrm{CRC}$, and that selective targeting of this protein may present a novel therapeutic strategy.

We demonstrated that lentiviral vectors carrying Jagged1shRNA-mediated Jagged1 silencing effectively blocked the Notch signaling in colon cancer cells, as reflected by a strong inhibition of Notch target genes. Downregulation of Jagged1 expression led to a reduced cell proliferation, colony formation, migration, and invasion. In vivo studies showed that xenograft tumors derived from Jagged1 knockdown cells displayed a significant reduction of growth speed and tumor volume, suggesting that direct downregulation of Jagged 1 has an antitumor effect in colon cancer.

Notch signaling influences cell proliferation depending on the cell type. For example, the Notch1 pathway has been found to have a tumor-suppressive effect on murine skin tumor and small cell lung cancer. $^{32,33}$ However, Notch1 appears to function as an oncogene in several human cancers. ${ }^{7-11}$ It has 

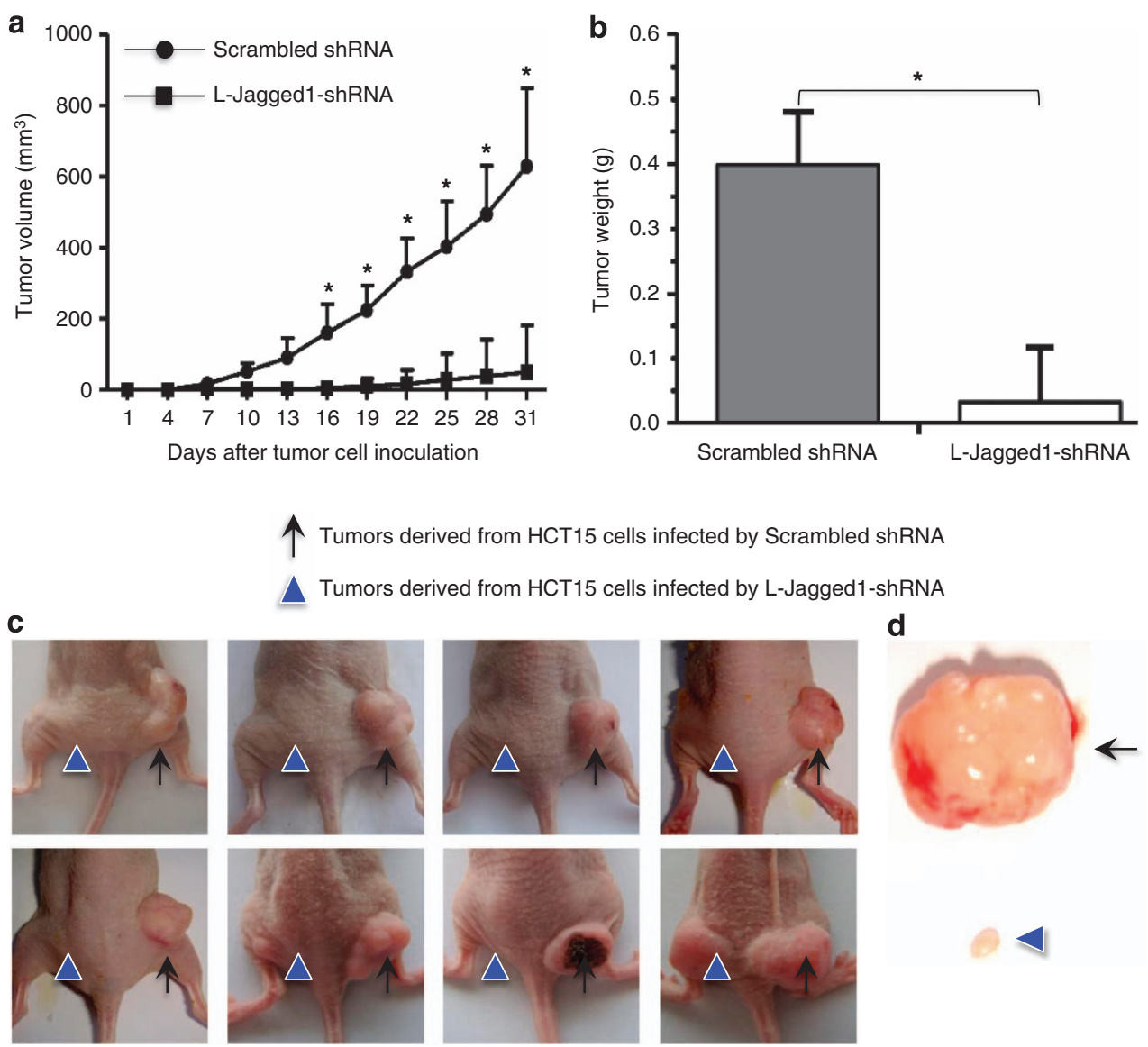

Figure 6 Effect of downregulation of Jagged1 by L-Jagged1-shRNA on the growth of xenograft tumors in nude mice. Mice $(n=8)$ were s.c. injected into the flanks with HCT15 cells stably infected with L-Jagged1-shRNA (left) or scrambled-shRNA (right). Tumor volumes were measured once every 3 days and tumor tissues were harvested 31 days after cell injection. The tumor growth curve was shown (a). The average tumor weight $(n=8)$ was measured after the mice were harvested (b). Representative tumorbearing mice (c) and the harvested xenograft tumor tissues (d) are shown. ${ }^{*} P<0.05$
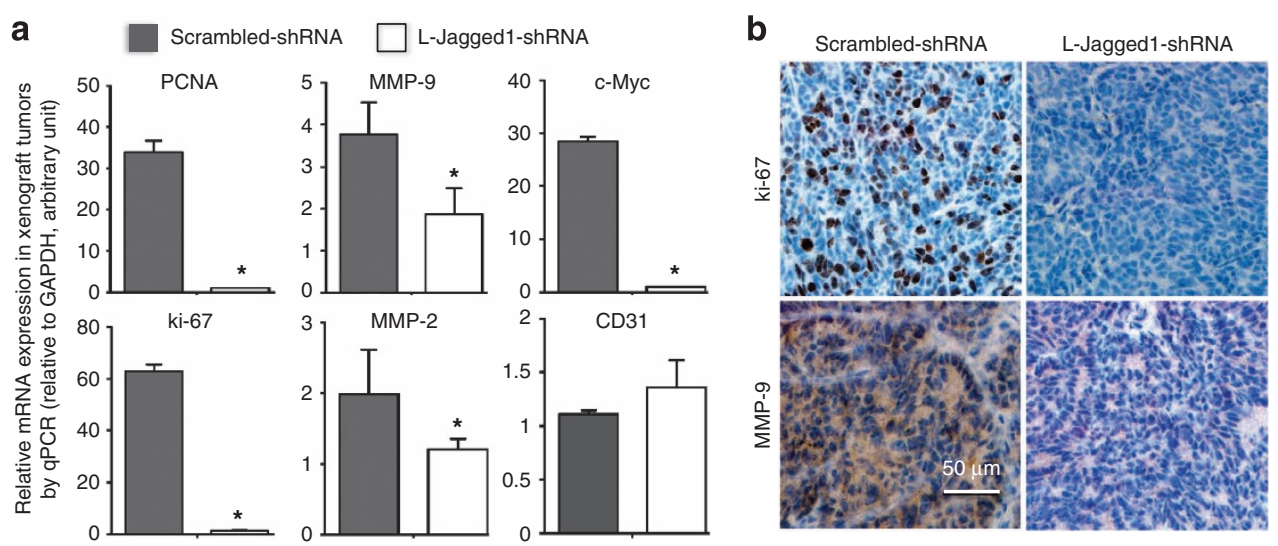

Figure 7 Expression of cell proliferation markers (PCNA, Ki-67, c-Myc) and metastatic markers (MMP-9, MMP-2), as well as the endothelial cell marker CD31 in xenograft tumors derived from HCT15 cells stably transfected with L-Jagged1-shRNA or scrambled-shRNA, was determined by qPCR (a). *P<0.05. Expression of cell proliferation marker Ki-67 and metastatic marker MMP-9 in the xenograft tumors was determined by immunohistochemistry (b)

been reported that Jagged1 is processed in a fashion similar to Notch1, ultimately resulting in the release of a nuclear targeted intracellular domain. ${ }^{34}$ Thus, Jagged1 is itself important in development and carcinogenesis. Here we found that downregulation of Jagged1 attenuated the growth of colon cancer cells and that this was associated with retarded cell cycle progression. The in vivo results also showed that Jagged1 knockdown dramatically impaired the tumorigenic growth of HCT15 cells. In agreement with this, we found a reduction in the number of Ki-67-positive cells in the tumors 
derived from Jagged1 knockdown cells compared with the ones from the control cells. Taken together, these observations suggested a positive role of Jagged1 in colon cancer cell growth and that Jagged1 may function as an oncogene in CRC.

The cell cycle is regulated by a series of checkpoints monitoring genomic integrity and ensuring that DNA replication proceeds in a coordinated manner. Aberrations in cell cycle progression occur in the majority of human malignancies. Different combinations of cyclin and CDK subunits operate at checkpoint controls during the cell cycle to integrate mitogenic and antiproliferative signals. Cyclin D1 and Cyclin $E$ have a critical role in controlling G1/S transition. ${ }^{35}$ The present study indicates that downregulation of Jagged 1 causes $G_{0} / G_{1}$ phase cell cycle arrest via a reduction of Cyclin D1 and Cyclin E levels, which appears to be the underlying mechanism in colon cancer cell growth inhibition. c-Myc is also important in cell cycle regulation and tumorigenesis. ${ }^{36,37}$ Jagged1 knockdown reduced c-Myc expression, so it might also contribute to the $\mathrm{G}_{0} / \mathrm{G}_{1}$ phase arrest. It was shown that regulation of the cell cycle by Notch signaling involves the coordination of different, and sometimes antagonizing, pathways in a highly cell contextdependent manner. Our observation was in line with the previous study by others ${ }^{20}$ that inhibition of Notch signaling by $\gamma$-secretase inhibitors resulted in $G_{1}$ phase arrest in colon cancer cells. However, downregulation of Jagged1 induced $S$ phase arrest in prostate cancer cells with reduced CDK2 kinase activity and increased p27 expression. ${ }^{38}$ On the other hand, Jagged1 knockdown results in an accumulation of ovarian cancer cells in the G2-M phase, ${ }^{39}$ suggesting the control of cell cycle progression by Jagged1 is cell-type specific.

Previous studies have shown that constitutively activated Notch signaling is involved in apoptosis resistance, and that abrogating Notch activation by the $\gamma$-secretase inhibitor, Notch1-siRNA, and DLL1-siRNA can induce apoptosis in human cancer cells. ${ }^{40,41}$ However, in the present study, a very mild apoptosis was detected in colon cancer cells upon Jagged1 knockdown both in vitro and in vivo. These findings are partially consistent with another report that Jagged1shRNA leads to significant cell growth inhibition in ovarian cancer cells but to a minor extent of apoptosis. ${ }^{42} \mathrm{We}$ speculate that several key members of the Notch cascade have different roles in apoptosis, and induction of apoptosis is not a major mechanism for targeting Jagged 1-mediated Notch signaling activation in CRC.

The Notch pathway was found to participate in many aspects of metastasis, the epithelial to mesenchymal transition, cell migration, invasion, and angiogenesis. ${ }^{43,44}$ $\gamma$-Secretase inhibitors were shown to reduce cell migration in different cancer cell lines. ${ }^{45} \mathrm{~A}$ recent report has shown that tumor-derived Jagged1 promotes bone metastasis of breast cancer. ${ }^{11}$ It is noteworthy that, in our study, downregulation of Jagged 1 significantly reduced the migration and invasion in colon cancer cells, and was associated with decreased expression of MMP-2 and MMP-9. MMP-2 and MMP-9 belong to the family of matrix metalloproteinases, and are present in invasive $\mathrm{CRC}^{46}$ The expression of these enzymes is known to be regulated by Notch signaling in cancer cells.
For instance, downregulation of Notch1 decreases MMP-9 levels in pancreatic cancer cells ${ }^{27}$ and prostate cancer cells. ${ }^{29}$ Here, we detected a decreased level of MMP-2 and MMP-9 upon Notch1 knockdown, suggesting that Jagged1 probably affects cell invasion via its action on the production of matrixdegrading enzymes. Although further studies are necessary to fully characterize the roles of Notch signaling in metastasis of $\mathrm{CRC}$, the inhibitory effect of Jagged1 knockdown on colon cancer cell migration is of particular interest, and Jagged1 may be a useful target molecule for developing new therapy against CRC.

Previous studies have shown that Jagged1 may have its own signaling function that is important to tumorigenesis independent of the canonical Notch pathway. ${ }^{39}$ Our study has demonstrated that Jagged1 targeting led to a marked reduction in the expression of Notch downstream target Hes1, suggesting that downregulation of Jagged1-mediated antitumor effect was at least partly through a Notchdependent mechanism in colon cancer cells. Since systemic inhibition of Notch signaling by $\gamma$-secretase inhibitors is associated with adverse effects such as gastrointestinal toxicity and liver injury, Jagged 1 may possibly constitute a promising target for CRC patients. It was noted that delivery of lentiviral vectors to patients may be practically impossible because protecting normal cells from being infected by the virus is a demanding task. Nanoparticles have been demonstrated to transport siRNA across cellular membranes and are biodegradable, biocompatible, and have low immunogenicity. ${ }^{47,48}$ Nanoparticles have been used to carry siRNAs against certain oncoproteins for the treatment of melanoma in a phase I clinical trial and showed no dose limiting cytotoxicity. ${ }^{48}$ Moreover, targeting Jagged 1 using this delivery system decreased cell viability and reversed taxane chemoresistance in ovarian cancer cells. ${ }^{39}$ Thus, our results establish a foundation on which targeted therapy can be developed.

In conclusion, we have shown that targeting Jagged1 inhibits proliferation, migration, and invasion of colon cancer cells in vitro, as well as suppresses the tumor growth and decreases tumor load in nude mice. With the ability to identify subsets of cancer patients with Jagged1 overexpression, antagonism of this signaling molecule could ultimately provide a useful therapeutic strategy for CRC.

\section{Materials and Methods}

Chemicals, reagents, and cell lines. Human colon cancer cell lines HCT15, HCT116, HT29, DLD1, and SW480 were purchased from the American Type Culture Collection (Manassas, VA, USA). Trizol, PCR-related reagents, and all cell culture-related materials were purchased from Invitrogen (Carlsbad, CA, USA). All the primary antibodies except anti-c-Myc (BD Biosciences, San Jose, CA, USA), anti-GAPDH (Abcam, Cambridge, UK) and anti-Ki-67 (DAKO, Glostrup, Denmark), and secondary antibodies were purchased from Santa Cruz Biotechnology (Santa Cruz, CA, USA).

Detection of Jagged1 in human colon cancer tissues by tissue microarray (TMA). Tissue microarray and fresh human CRC tissues and matched non-cancerous colonic tissues were described in detail in our previous publication. ${ }^{49}$ These cancer tissues were largely obtained from patients who underwent surgical operations for CRC. All cancer tissues, matched noncancerous tissues, and TMAs were provided by M Ebert of the Technical University, Munich, Germany. In the current study, all 24 tissues were adenocarcinomas, and classified into well $(n=3)$, moderately $(n=18)$, and 
poorly $(n=3)$ differentiated. Patient ages ranged from 49 years to 77 years (mean, 64 years), consisting of 16 males and 8 females.

The expression of Jagged1 was detected by immunohistochemistry in TMA slides, as reported in our previous publications. ${ }^{49}$ The slides were evaluated by a senior pathologist (KW Chan), and the quantitative score was generated as previously reported. ${ }^{49,50}$ The study was approved by the institutional human ethics committee of the relevant institutions.

Construction of recombinant lentiviral vectors. A 21-mer oligonucleotide sequence from nucleotide 1496 to 1516 of the Jagged 1 human mRNA sequence was used. The hairpin sense and antisense sequences were as follows: 5'-CCGGTGGTATTCAGGACCCAACTGTGCTCGAGCACAGTTGGGTCCTGAAT ACCTTTTTG- $3^{\prime}$ and 5'-AATTCAAAAAGGTATTCAGGACCCAACTGTGCTCGAG CACAGTTGGGTCCTGAATACCA-3'. The oligonucleotides were annealed and cloned into the Agel/EcoR1 sites of the shRNA vector pLKO.1-puro (Addgene, Cambridge, MA, USA). Expression of the shRNA was controlled by the human U6 promoter. To produce lentiviral particles, the constructed Jagged1-shRNA or scrambled-shRNA plasmid was co-transfected with psPAX2 packaging plasmid and pMD2.G envelope plasmid into 293T cells using X-tremeGENE HP DNA transfection Reagent (Roche, Basel, Switzerland). The titer of the purified virus was determined in 293T cells by the serial dilution method.

Cell culture and construction of Jagged1 knockdown cells. Human colon cancer cell lines HCT116, HCT15, HT29, DLD1, and SW480 were cultured in RPMl1640 medium supplemented with 10\% FBS and $1 \%$ Pen-Strep at $37^{\circ} \mathrm{C}$ with $5 \% \mathrm{CO}_{2}$. Cells were passaged in our laboratory for lesser than 6 months after resuscitation. Mycoplasma contamination was tested by PCR during culture, and no additional authentication was done as cells came from national repositories. Cells were grown to $70-80 \%$ confluence, and incubated with the constructed lentiviral vectors for $24 \mathrm{~h}$ in growth medium containing $8 \mu \mathrm{g} / \mathrm{ml}$ of polybrene (Santa Cruz). Seventy-two hours after infection, the medium was changed to fresh RPMl1640 containing $10 \mu \mathrm{g} / \mathrm{ml}$ puromycin (Santa Cruz). Puromycin-resistant colonies were used for subsequent studies.

Cell proliferation and colony formation assay. Cell proliferation was determined by $\left[{ }^{3} \mathrm{H}\right]$-thymidine incorporation assay, as we previously reported. ${ }^{51}$ Colony formation assay was used to determine the anchorage-dependent growth property of the cells and was performed, as described previously. ${ }^{52}$ Briefly, Jagged1 knockdown and the control cells were plated in six-well plates at a density of 3000 cells/well. Plates were incubated for 7 days, and cell colonies were visualized by staining with crystal violet and macroscopically counted.

Cell cycle analysis. Cell cycle was detected by propidium iodide (PI) staining and flow cytometric analysis. Briefly, cells were washed with PBS, fixed with $70 \%$ ethanol at $4{ }^{\circ} \mathrm{C}$ overnight. After washing with PBS, cells were re-suspended in $50 \mu \mathrm{g} / \mathrm{ml}$-of PI containing 0.5\% Triton-X 100 and $100 \mu \mathrm{g} / \mathrm{ml}$ of RNase A and incubated in the dark at $37^{\circ} \mathrm{C}$ for $30 \mathrm{~min}$. The cells were then analyzed for DNA content using a FACS Calibur Cytometer (BD Biosciences). A minimum of 10000 events per sample was acquired and subsequently analyzed with ModFit software (Verity Software House, Topsham, ME, USA).

Cell migration and invasion assay. Cell migration was analyzed by a conventional wound-healing assay, as described previously. ${ }^{53}$ Briefly, Jagged1shRNA or scrambled-shRNA stably infected cells were grown in six-well plates to complete confluence. Wound injury was made with the tip of a sterile micropipette, and detached cells were removed by washing with PBS. Cells were then incubated and allowed to migrate for up to $72 \mathrm{~h}$. Photographs were taken and migration index was calculated as follows: migration index $=$ [(initial wound width-width of wound at time point tested)/initial wound width] $\times 100 \%$.

In vitro invasion assay was performed using a 24-well transwell insert ( $8 \mu \mathrm{m}$ pore size) pre-coated with $\mathrm{BD}$ Matrigel matrix (BD Biosciences) according to the manufacturer's instruction. Cells transfected with Jagged1-shRNA or scrambledshRNA were seeded into the upper chamber in serum-free medium at a density of 50000 cells/well. The medium containing $10 \%$ FBS was placed in the lower chamber to act as a chemoattractant, and cells were further incubated for the indicated time. Non-invading cells were removed from the upper chamber and the invaded cells remaining on the lower surface of the insert were fixed and stained with crystal violet. Cells were quantified as the average number of cells found in five random microscopic fields in three independent inserts.

Western blot and qPCR assays. Cells were lysed in RIPA buffer (Sigma, St. Louis, MO, USA) supplemented with $1 \%$ protease inhibitor cocktail. Proteins from the cell lysate were separated on a 10\% SDS-PAGE and transferred onto polyvinylidenedifluoride membranes (Millipore, Billerica, MA, USA). After blocking, the membranes were probed with appropriate antibodies and detected with Enhanced Chemiluminescence Assay (Pierce Biotechnology, Rockford, IL, USA).

For the gene expression at the mRNA level, total RNA was extracted, quantified, and converted to CDNA, and GPCR was performed using SYBR Green (Applied Biosystems, Foster City, CA, USA) system. The sequences of primers used in qPCR were as follows: JAGGED1 forward: $5^{\prime}$-TCGGGTCAGTTCGAGTTGGA-3' and reverse: 5'-CGTTCACGTTCTGCATGGAC-3'; PCNA forward: 5'-GCGTG AACCTCACCAGTATGT- $3^{\prime}$ and reverse: $5^{\prime}$-TCTTCGGCCCTTAGTGTAATGAT-3'; Ki67 forward: $5^{\prime}$-ACGCCTGGTTACTATCAAAAGG-3' and reverse: $5^{\prime}$-CAGACC CATTTACTTGTGTTGGA-3'; c-MYC forward: 5'-GGCTCCTGGCAAAAGGTCA-3' and reverse: $5^{\prime}$-CTGCGTAGTTGTGCTGATGT-3'; MMP2 forward: 5'-TACAGGAT CATTGGCTACACACC-3' and reverse: $5^{\prime}$-GGTCACATCGCTCCAGACT-3'; MMP9 forward: $5^{\prime}$-TGTACCGCTATGGTTACACTCG-3' and reverse: $5^{\prime}$-GGCAGGGAC AGTTGCTTCT-3'; HES1 forward: 5'-ACGTGCGAGGGCGTTAATAC-3' and reverse: $5^{\prime}$-GGGGTAGGTCATGGCATTGA-3'; CD31 forward: $5^{\prime}$-ACAGTGTTGA CATGAAGAGCC- $3^{\prime}$ and reverse: $5^{\prime}$-TGTAAAACAGCACGTCATCCTT-3';GAPDH forward: $5^{\prime}$-CTGGGCTACACTGAGCACC-3' and reverse: $5^{\prime}$-AAGTGGTCGTTG AGGGCAATG-3'. The relative quantification of mRNA was normalized to GAPDH.

Xenograft tumorigenesis in nude mice. Athymic nude mice (BALB/C nu/nu) (male, 6-8-week-old) were purchased from the Academy of Military Medical Science (Beijing, China), and were acclimated for 7 days in the laboratory before experimentation. To establish xenograft tumors, $1 \times 10^{7}$ Jagged 1 knockdown and the control HCT15 cells were injected subcutaneously into the dorsal flanks of the mice. Tumor volumes were calculated every 3 days according to the following formula: volume $\left(\mathrm{mm}^{3}\right)=\left[(\text { shortest diameter })^{2} \times\right.$ longest diameter $] / 2$. On the day of harvest the tumor tissues were excised, size was measured and weight was determined. Harvested tumor tissues were either snap frozen for RNA analysis or fixed in $10 \%$ neutral formalin for histology assay. All animal experiments were approved by the Committee on the Use of Live Animals in Teaching and Research of the Tianjin Medical University.

Statistical analysis. Data were expressed as mean \pm S.D. Student's independent two-sample t-tests were used for comparisons. A $P$ value of $<0.05$ was considered statistically significant.

\section{Conflict of Interest}

The authors declare no conflict of interest.

Acknowledgements. This work was partially supported by research grants from (1) the Ministry of Science and Technology of China (Grant No. 2012CB932503 and 2011CB933100); (2) National Natural Science Foundation of China (Grant No. 81072019, 91029705, 81172864, and 81272317); (3) Drs. L Qiao, G Wilson, and J George's work was supported by the Robert W. Storr Bequest to the Sydney Medical Foundation, University of Sydney, a National Health and Medical Research Council of Australia (NHMRC) Program Grant No. 1053206 and a Project grant (APP1047417), a Cancer Council NSW grant (APP1070076), and the Sydney West Translational Cancer Research Centre Partner Program funded by the Cancer Institute NSW. Dr. L Qiao is supported by the Career Development and Support Fellowship Future Research Leader Grant of the NSW Cancer Institute, Australia (Grant ID: 08/FRL/1-04).

1. Jemal A, Bray F, Center MM, Ferlay J, Ward E, Forman D. Global cancer statistics. CA Cancer J Clin 2011; 61: 69-90.

2. Center MM, Jemal $A$, Smith RA, Ward $E$. Worldwide variations in colorectal cancer. CA Cancer J Clin 2009; 59: 366-378.

3. Center $M M$, Jemal $A$, Ward $E$. International trends in colorectal cancer incidence rates. Cancer Epidemiol Biomarkers Prev 2009; 18: 1688-1694. 
4. Davies JM, Goldberg RM. Treatment of metastatic colorectal cancer. Semin Oncol 2011; 38: $552-560$

5. Fortini ME. Notch signaling: the core pathway and its posttranslational regulation. Dev Cell 2009; 16: 633-647.

6. Artavanis-Tsakonas S, Rand MD, Lake RJ. Notch signaling: cell fate control and signal integration in development. Science 1999; 284: 770-776.

7. Santagata S, Demichelis F, Riva A, Varambally S, Hofer MD, Kutok JL et al. JAGGED1 expression is associated with prostate cancer metastasis and recurrence. Cancer Res 2004; 64: 6854-6857.

8. Purow BW, Haque RM, Noel MW, Su Q, Burdick MJ, Lee J et al. Expression of Notch-1 and its ligands, Delta-like-1 and Jagged-1, is critical for glioma cell survival and proliferation. Cancer Res 2005; 65: 2353-2363.

9. Reedijk M, Odorcic S, Chang L, Zhang H, Miller N, McCready DR et al. High-level coexpression of JAG1 and NOTCH1 is observed in human breast cancer and is associated with poor overall survival. Cancer Res 2005; 65: 8530-8537.

10. Lin JT, Chen MK, Yeh KT, Chang CS, Chang TH, Lin CY et al. Association of high levels of Jagged-1 and Notch-1 expression with poor prognosis in head and neck cancer. Ann Surg Oncol 2010; 17: 2976-2983.

11. Sethi N, Dai X, Winter CG, Kang Y. Tumor-derived JAGGED1 promotes osteolytic bone metastasis of breast cancer by engaging notch signaling in bone cells. Cancer Cell 2011; 19: 192-205

12. Lu J, Ye X, Fan F, Xia L, Bhattacharya R, Bellister S et al. Endothelial cells promote the colorectal cancer stem cell phenotype through a soluble form of Jagged-1. Cancer Cell 2013; 23: 171-185.

13. Kim MH, Kim HB, Yoon SP, Lim SC, Cha MJ, Jeon YJ et al. Colon cancer progression is driven by APEX1-mediated upregulation of Jagged. J Clin Invest 2013; 123: 3211-3230.

14. Qiao L, Wong BC. Role of Notch signaling in colorectal cancer. Carcinogenesis 2009; 30 1979-1986.

15. vanEs JH, van Gijn ME, Riccio O, van den Born M, Vooijs M, Begthel $\mathrm{H}$ et al. Notch gamma-secretase inhibition turns proliferative cells in intestinal crypts and adenomas into goblet cells. Nature 2005; 435: 959-963.

16. Rodilla V, Villanueva A, Obrador-Hevia A, Robert-Moreno A, Fernández-Majada V, Grilli A et al. Jagged1 is the pathological link between Wnt and Notch pathways in colorectal cancer. Proc Natl Acad Sci USA 2009; 106: 6315-6320.

17. Veenendaal LM, Kranenburg O, Smakman N, Klomp A, BorelRinkes IH, van Diest PJ. Differential Notch and TGFbeta signaling in primary colorectal tumors and their corresponding metastases. Cell Oncol 2008; 30: 1-11

18. Sikandar SS, Pate KT, Anderson S, Dizon D, Edwards RA, Waterman ML et al. NOTCH signaling is required for formation and self-renewal of tumor-initiating cells and for repression of secretory cell differentiation in colon cancer. Cancer Res 2010; 70: 1469-1478.

19. Sonoshita M, Aoki M, Fuwa H, Aoki K, Hosogi H, Sakai Y et al. Suppression of colon cance metastasis by Aes through inhibition of Notch signaling. Cancer Cell 2011; 19: 125-137.

20. Guilmeau S, Flandez M, Mariadason JM, Augenlicht LH. Heterogeneity of Jagged1 expression in human and mouse intestinal tumors: implications for targeting Notch signaling. Oncogene 2010; 29: 992-1002.

21. Guilmeau S. Notch signaling and intestinal cancer. Adv Exp Med Biol 2012; 727: 272-288.

22. Akiyoshi T, Nakamura M, Yanai K, Nagai S, Wada J, Koga K et al. Gamma-secretase inhibitors enhance taxane-induced mitotic arrest and apoptosis in colon cancer cells Gastroenterology 2008; 134: 131-144.

23. Teicher BA. Antiangiogenic agents and targets: a perspective. Biochem Pharmacol 2011; 81: $6-12$.

24. Jensen J, Pedersen EE, Galante P, Hald J, Heller RS, Ishibashi M et al. Control of endodermal endocrine development by Hes-1. Nat Genet 2000; 24: 36-44.

25. Guilmeau S, Flandez M, Bancroft L, Sellers RS, Tear B, Stanley P et al. Intestinal deletion of Pofut1 in the mouse inactivates notch signaling and causes enterocolitis. Gastroenterology 2008; 135: 849-860.

26. Pellegrinet L, Rodilla V, Liu Z, Chen S, Koch U, Espinosa L et al. Dll1- and dll4-mediated notch signaling are required for homeostasis of intestinal stem cells. Gastroenterology 2011; 140: 1230-1240.

27. Wang Z, Banerjee S, Li Y, Rahman KM, Zhang Y, Sarkar FH. Down-regulation of notch-1 inhibits invasion by inactivation of nuclear factor-kappaB, vascular endothelial growth factor, and matrix metalloproteinase-9 in pancreatic cancer cells. Cancer Res 2006; 66 : 2778-2784.

28. Zhang P, Yang Y, Zweidler-McKay PA, Hughes DP. Critical role of notch signaling in osteosarcoma invasion and metastasis. Clin Cancer Res 2008; 14: 2962-2969.

29. Bin Hafeez B, Adhami VM, Asim M, Siddiqui IA, Bhat KM, Zhong W et al. Targeted knockdown of Notch1 inhibits invasion of human prostate cancer cells concomitant with inhibition of matrix metalloproteinase-9 and urokinase plasminogen activator. Clin Cance Res 2009; 15: 452-459.

30. Gao J, Liu J, Fan D, Xu H, Xiong Y, Wang Y et al. Up-regulated expression of Notch1 and Jagged1 in human colon adenocarcinoma. Pathol Biol (Paris) 2011; 59: 298-302.
31. Jin HY, Zhang HY, Wang X, Xu J, Ding Y. Expression and clinical significance of Notch signaling genes in colorectal cancer. Tumour Biol 2012; 33: 817-824.

32. Nicolas M, Wolfer A, Raj K, Kummer JA, Mill P, van Noort M et al. Notch1 functions as a tumor suppressor in mouse skin. Nat Genet 2003; 33: 416-421.

33. Sriuranpong V, Borges MW, Ravi RK, Arnold DR, Nelkin BD, Baylin SB et al. Notch signaling induces cell cycle arrest in small cell lung cancer cells. Cancer Res 2001; 61: 3200-3205

34. LaVoie MJ, Selkoe DJ. The Notch ligands, Jagged and Delta, are sequentially processed by alpha-secretase and presenilin/gamma-secretase and release signaling fragments. J Biol Chem 2003; 278: 34427-34437.

35. Nasmyth K. Viewpoint: putting the cell cycle in order. Science 1996; 274: 1643-1645.

36. Eisenman RN. Deconstructing myc. Genes Dev 2001; 15: 2023-2030.

37. Nesbit CE, Tersak JM, Prochownik EV. MYC oncogenes and human neoplastic disease. Oncogene 1999; 18: 3004-3016.

38. Zhang Y, Wang Z, Ahmed F, Banerjee S, Li Y, Sarkar FH. Down-regulation of Jagged-1 induces cell growth inhibition and $\mathrm{S}$ phase arrest in prostate cancer cells. Int J Cancer 2006; 119: 2071-2077.

39. Steg AD, Katre AA, Goodman B, Han HD, Nick AM, Stone RL et al. Targeting the notch ligand JAGGED1 in both tumor cells and stroma in ovarian cancer. Clin Cancer Res 2011; 17: $5674-5685$.

40. Rosati E, Sabatini R, Rampino G, Tabilio A, Di lanni M, Fettucciari K et al. Constitutively activated Notch signaling is involved in survival and apoptosis resistance of B-CLL cells. Blood 2009; 113: 856-865.

41. Lin L, Mernaugh R, Yi F, Blum D, Carbone DP, Dang TP. Targeting specific regions of the Notch3 ligand-binding domain induces apoptosis and inhibits tumor growth in lung cancer. Cancer Res 2010; 70: 632-638.

42. Choi JH, Park JT, Davidson B, Morin PJ, Shih leM, Wang TL. Jagged-1 and Notch3 juxtacrine loop regulates ovarian tumor growth and adhesion. Cancer Res 2008; 68: 5716-5723.

43. Bailey JM, Singh PK, Hollingsworth MA. Cancer metastasis facilitated by developmental pathways: Sonic hedgehog, Notch, and bone morphogenic proteins. J Cell Biochem 2007; 102: 829-839.

44. Dufraine J, Funahashi Y, Kitajewski J. Notch signaling regulates tumor angiogenesis by diverse mechanisms. Oncogene 2008; 27: 5132-5137.

45. Sahlgren C, Gustafsson MV, Jin S, Poellinger L, Lendahl U. Notch signaling mediates hypoxia-induced tumor cell migration and invasion. Proc Natl Acad Sci USA 2008; 105: 6392-6397.

46. Waas ET, Wobbes T, Lomme RM, DeGroot J, Ruers T, Hendriks T. Matrix metalloproteinase 2 and 9 activity in patients with colorectal cancer liver metastasis. Br J Surg 2003; 90: 1556-1564.

47. Burnett JC, Rossi JJ, Tiemann K. Current progress of siRNA/shRNA therapeutics in clinical trials. Biotechnol J 2011; 6: 1130-1146.

48. Davis ME, Zuckerman JE, Choi CH, Seligson D, Tolcher A, Alabi CA et al. Evidence of RNAi in humans from systemically administered siRNA via targeted nanoparticles. Nature 2010; 464: 1067-1070.

49. Qiao L, Dai Y, Gu Q, Chan KW, Ma J, Lan HY et al. Loss of XIAP sensitizes colon cancer cells to PPARgamma independent antitumor effects of troglitazone and 15-PGJ2. Cancer Lett 2008; 268: 260-271.

50. Qiao L, Dai Y, Gu Q, Chan KW, Zou B, Ma J et al. Down-regulation of X-linked inhibitor of apoptosis synergistically enhanced peroxisome proliferator-activated receptor gamma ligand-induced growth inhibition in colon cancer. Mol Cancer Ther 2008; 7: 2203-2211.

51. Dai Y, Qiao L, Chan KW, Yang M, Ye J, Ma J et al. Peroxisome proliferator-activated receptor-gamma contributes to the inhibitory effects of Embelin on colon carcinogenesis. Cancer Res 2009; 69: 4776-4783.

52. Tu SP, Jiang XH, Lin MC, Cui JT, Yang Y, Lum CT et al. Suppression of survivin expression inhibits in vivo tumorigenicity and angiogenesis in gastric cancer. Cancer Res 2003; 63: 7724-7732.

53. Qiao L, Gu Q, Dai Y, Shen Z, Liu X, Qi R et al. XIAP-associated factor 1 (XAF1) suppresses angiogenesis in mouse endothelial cells. Tumour Biol 2008; 29: 122-129.

(c) (i) $\odot$ Cell Death and Disease is an open-access journa published by Nature Publishing Group. This work is licensed under a Creative Commons Attribution-NonCommercialNoDerivs 3.0 Unported License. The images or other third party material in this article are included in the article's Creative Commons license, unless indicated otherwise in the credit line; if the material is not included under the Creative Commons license, users will need to obtain permission from the license holder to reproduce the material. To view a copy of this license, visit http://creativecommons.org/licenses/ by-nc-nd/3.0/ 